01

\title{
Математическое моделирование образования гистонового октамера
}

\author{
(C) Т.В. Кошлан, ${ }^{1}$ К.Г. Куликов ${ }^{2, \text { ฯ }}$ \\ ${ }^{1}$ Санкт-Петербургский государственный университет, \\ 199034 Санкт-Петербург, Россия \\ ${ }^{2}$ Санкт-Петербургский политехнический университет Петра Великого, \\ 195251 Санкт-Петербург, Россия \\ ฯ e-mail: kulikov.kirill.g@gmail.com
}

(Поступило в Редакцию 21 сентября 2016 г.)

Разработана физическая модель взаимодействия белковых молекул и их способности образовывать сложные биологические комплексы для случая in vitro в растворе одновалентной соли. Изучены их реакционные способности с использованием методов электростатики на примере пошагового образования гистонового октамера из белков Н2А, Н2B, Н3, Н4. Для анализа способности белковых молекул образовывать соединения, проанализирована матрица потенциальной энергии взаимодействия белковых молекул в растворах с различной концентрацией одновалентной соли.

DOI: 10.21883/JTF.2017.05.44437.2041

\section{Введение}

Настоящая работа посвящена разработке математической модели, которая позволит описать поведение сложных биологических комплексов: димеров, тетрамеров, гексамеров, октамеров in vitro в растворах с различной концентрацией одновалентной соли при образовании гистонового ядра нуклеосомы.

В настоящей работе мы изучали стабильность биологических комплексов в растворах с различной ионной силой с учетом эффекта экранирования заряженных аминокислотных остатков: лизина, аргинина, гистидина, аспарагиновой кислоты, глутаминовой кислоты.

Следует отметить, что в представленной работе не учитывалось поведение неполярных, полярных, ароматических аминокислот в растворах с различной ионной силой, так же наша модель не учитывала конформацию белковых молекул.

Отметим ряд работ, связанных с анализом формирования ядра нуклеосомы. В [1] изучали кристаллические структуры четырех мутантных нуклеосом, в которых один из четырех гистонов Н2А, Н2B, Н3, Н4 был без Nконцевого участка. На основании проведенных исследований были получены данные о влиянии данного участка у различных гистонов на стабильность нуклеосомы.

Работа [2] посвящена вопросу устойчивости биологических комплексов $(\mathrm{H} 2 \mathrm{~A}-\mathrm{H} 2 \mathrm{~B})$ и $(\mathrm{H} 3-\mathrm{H} 4)_{2}$ in vitro при различных физических условиях в присутствии солей и денатурирующих агентов. Показано, что тетрамер $(\mathrm{H} 3-\mathrm{H} 4)_{2}$ менее устойчив, чем димер (Н2A-H2B).

В [3] приведен анализ внутри нуклеосомных взаимодействий между различными вариантами гистонов и указаны участки связывания между гистонами при образовании нуклеосомы.

Однако следует отметить, что в приведенных работах не приведен критерий для количественной оценки сил электростатического взаимодействия между белковыми единицами, приводящими к сборке или диссоциации гистонового октамера при различных концентрациях одновалентной соли.

Настоящая работа в отличие от вышеприведенных позволяет дать количественную оценку сил электростатического взаимодействия между гистоновыми белками в растворах с различной концентрацией одновалентной соли с учетом эффекта экранирования заряженных аминокислотных остатков.

Работа состоит из нескольких частей: в первой части описана структура гистонового ядра, основные принципы его формирования. Во второй части выведено уравнение для расчета эффекта экранирования. В третей части рассмотрена задача пошагового образования гистонового октамера с учетом эффекта экранирования в солевом растворе с различной концентрацией одновалентной соли.

\section{1. Общие принципы формирования гистонового октамера}

Нуклеосома представляет собой октамер белков гистонов, который несет на себе 145-147 пар нуклеотидов ДНК, которая закручивается вокруг гистонового ядра, образуя 1.65 витка левозакрученной суперспирали. В ядро нуклеосомы входит четыре семейства гистонов Н3 и Н4, Н2А и Н2В, каждый из которых представлен дважды. Восемь гистонов нуклеосомы собраны в четыре гетеродимера [3]: два (Н2A-H2B) и два (H3-H4).

Процесс сборки октамера начинается с присоединения димера (H3-H4) к такому же димеру (H3-H4), при этом образуется тетрамер $(\mathrm{H} 3-\mathrm{H} 4)_{2}$, далее к тетрамеру присоединяется димер (Н2A-H2B) в результате образуется гексамер (H3-H4) 2 (H2A-H2B). К гексамеру присоединяется еще один димер (Н2A-H2B) и завершает образование биологического комплекса формирование октамера $(\mathrm{H} 3-\mathrm{H} 4)_{2}(\mathrm{H} 2 \mathrm{~A}-\mathrm{H} 2 \mathrm{~B})_{2}$. 
В представленной работе построена физическая модель, которая моделирует стадии образования гистонового октамера для случая in vitro при различных концентрациях одновалентной соли, а именно [4]:

- образование димеров (Н2A-H2B) и (Н3-H4),

- образование тетрамера $(\mathrm{H} 3-\mathrm{H} 4)_{2}$,

- образование гексамера $(\mathrm{H} 3-\mathrm{H} 4)_{2}(\mathrm{H} 2 \mathrm{~A}-\mathrm{H} 2 \mathrm{~B})$,

- образование октамера $(\mathrm{H} 3-\mathrm{H} 4)_{2}(\mathrm{H} 2 \mathrm{~A}-\mathrm{H} 2 \mathrm{~B})_{2}$.

Нами были промоделированы следующие взаимодействия:

- образование димеров из мономеров $(\mathrm{H} 3+\mathrm{H} 4) \rightarrow$ $\rightarrow(\mathrm{H} 3-\mathrm{H} 4),(\mathrm{H} 2 \mathrm{~A}+\mathrm{H} 2 \mathrm{~B}) \rightarrow(\mathrm{H} 2 \mathrm{~A}-\mathrm{H} 2 \mathrm{~B})$,

- связывание димеров в тетрамеры $(\mathrm{H} 3-\mathrm{H} 4)+$ $+(\mathrm{H} 3-\mathrm{H} 4) \rightarrow(\mathrm{H} 3-\mathrm{H} 4)_{2}$,

- присоединение димера к тетрамеру с образованием гексамера $(\mathrm{H} 3-\mathrm{H} 4)_{2}+(\mathrm{H} 2 \mathrm{~A}-\mathrm{H} 2 \mathrm{~B}) \rightarrow$ $\rightarrow(\mathrm{H} 3-\mathrm{H} 4)_{2}(\mathrm{H} 2 \mathrm{~A}-\mathrm{H} 2 \mathrm{~B})$

- присоединение димера к гексамеру с образованием гистонового октамера $(\mathrm{H} 3-\mathrm{H} 4)_{2}(\mathrm{H} 2 \mathrm{~A}-\mathrm{H} 2 \mathrm{~B})+$ $+(\mathrm{H} 2 \mathrm{~A}-\mathrm{H} 2 \mathrm{~B}) \rightarrow(\mathrm{H} 3-\mathrm{H} 4)_{2}(\mathrm{H} 2 \mathrm{~A}-\mathrm{H} 2 \mathrm{~B})_{2}$.

Отметим, что биологические объекты взаимодействуют в растворе, который может обладать различной ионной силой, т. е. содержать различные растворенные ионы. В таких биологических системах большое значение имеют взаимодействия между ионами, которые сильно зависят от ионной силы раствора. Данная величина является мерой интенсивности электрического поля, создаваемого ионами в растворе. Чтобы учесть влияние величины ионной силы в растворе на устойчивость изучаемых нами биологических соединений, мы использовали теорию Гуи-Чепмена для расчета экранирующего потенциала заряженной аминокислотной последовательности белка при различных концентрациях одновалентной соли в растворе с биологическими объектами.

\section{1. Учет эффекта экранирования в солевом растворе}

Для учета механизма формирования компенсирующего слоя ионов в растворе (эффект экранирования), который формируется за счет сил электростатического притяжения к распределенному на поверхности заряду, мы использовали теорию Гуи-Чепмена [5-7]. В данной теории ионы электролита описываются точечными зарядами обоих знаков в водной среде с определенной диэлектрической проницаемостью.

Если энергия ионов в поле притяжения к поверхности заряда порядка $k T$ (где $k-$ постоянная Больцмана, $T$ - абсолютная температура), то тепловое движение должно сделать такой слой диффузным. Таким образом, пространственное распределение противоионов (ионы, имеющие противоположный заряд) определяется тем, что они находятся в состоянии теплового движения и одновременно притягиваются к поверхностному заряду, в результате чего образуют диффузный слой определенной протяженности. Отметим, что протяженность при низких концентрациях электролита может быть весьма значительной.

Напряженность электрического поля в двойном слое должна монотонно убывать при удалении от заряженной поверхности, поскольку ее заряд экранируется зарядом противоионов, размещенных между данной удаленной точкой и заряженной поверхностью.

У внешней границы двойного электрического слоя электрическое поле должно исчезать. Таким образом, единственной переменной, от которой зависит функция спада потенциала, является расстояние от заряженной поверхности. Отметим, что радиус частицы сонаправлен с вектором расстояния от заряженной поверхности.

В соответствии с этой моделью функции электрического потенциала и соответствующего среднего распределения заряда вычисляются в окрестности заряженной поверхности. Расчет электрического двойного слоя для заряженной поверхности сферы был выполнен для пяти заряженных аминокислот: аспарагиновая кислота $(D)$, глутаминовая кислота $(E)$, лизин $(K)$, аргинин $(R)$, гистидин $(H)$.

Запишем уравнение Пуассона для плоской поверхности

$$
\frac{d^{2} \varphi(x)}{d x^{2}}=-\frac{\rho}{\varepsilon \varepsilon_{0}},
$$

где $\rho$ - плотность заряда, определенная на расстоянии $x$ от поверхности и $\varphi(x)$ - потенциал, $\varepsilon$ - диэлектрическая проницаемость среды, $\varepsilon_{0}-$ электрическая постоянная.

В соответствии с [5-7] запишем общую плотность заряда на единицу объема для определенного иона

$$
\rho=\sum_{i=1}^{N} n_{i} z_{i} e=\sum_{i=1}^{N} n_{i}^{0} z_{i} e \exp \left[\frac{-z_{i} e \varphi(x)}{k T}\right],
$$

где $n_{i}^{0}$ - концентрация иона в растворе, $e-$ заряд электрона, $z$ - заряд иона.

Комбинируя (1) и (2), мы получаем уравнение Пуассона-Больцмана

$$
\frac{d^{2} \varphi(x)}{d x^{2}}=-\frac{e}{\varepsilon \varepsilon_{0}} \sum_{i=1}^{N} n_{i}^{0} z_{i} \exp \left[\frac{-z_{i} e \varphi(x)}{k T}\right] .
$$

Приведенное уравнение необходимо дополнить граничными условиями [7]

$$
\varphi(0)=\varphi_{0},\left.\quad \varphi\right|_{x \rightarrow \infty}=0 .
$$

Умножив уравнение (3) на $\frac{d \varphi}{d x}$ слева и справа, получим

$$
\begin{gathered}
\frac{1}{2} \frac{d}{d x}\left(\frac{d \varphi}{d x}\right)^{2}=-\frac{e}{\varepsilon \varepsilon_{0}} \sum_{i=1}^{N} n_{i}^{0} z_{i} \exp \left[\frac{-z_{i} e \varphi(x)}{k T}\right]\left(\frac{d \varphi}{d x}\right) \\
\frac{1}{2} \frac{d}{d x}\left(\frac{d \varphi}{d x}\right)^{2}=\frac{d}{d x} \frac{k T}{\varepsilon \varepsilon_{0}} \sum_{i=1}^{N} n_{i}^{0} \exp \left[\frac{-z_{i} e \varphi(x)}{k T}\right]
\end{gathered}
$$


После интегрирования уравнения (5) и с учетом обращения в нуль производной потенциала вдали от поверхности, с помощью которой определяется постоянная интегрирования, имеем

$$
\begin{aligned}
& \frac{1}{2}\left(\frac{d \varphi}{d x}\right)^{2}=\frac{k T}{\varepsilon \varepsilon_{0}} \sum_{i=1}^{N} n_{i}^{0} \exp \left[\left[\frac{-z_{i} e \varphi(x)}{k T}\right]-1\right] \\
& \frac{d \varphi}{d x}= \pm\left[\frac{2 k T}{\varepsilon \varepsilon_{0}} \sum_{i=1}^{N} n_{i}^{0} \exp \left[\left[\frac{-z_{i} e \varphi(x)}{k T}\right]-1\right]\right]^{1 / 2}
\end{aligned}
$$

Отметим, что полученное уравнение (7) можно интегрировать в случае произвольных потенциалов поверхности, но только для симметричного электролита

$$
z_{+}=-z_{-}=z, \quad n_{+}^{0}=n_{-}^{0}=n^{0}
$$

Преобразуем выражение, входящее в правую часть уравнения (7):

$$
\sum_{i=1}^{N} n_{i}^{0} \exp \left[\left[\frac{-z_{i} e \varphi(x)}{k T}\right]-1\right]
$$

следующим образом:

$$
\begin{aligned}
\sum_{i=1}^{N} n_{i}^{0} \exp \left[\left[\frac{-z_{i} e \varphi(x)}{k T}\right]-1\right] & =2 n^{0}\left[\operatorname{ch}\left[\frac{z e \varphi(x)}{k T}\right]-1\right] \\
& =4 n^{0} \operatorname{sh}^{2}\left[\frac{z e \varphi(x)}{2 k T}\right]
\end{aligned}
$$

Тогда с учетом выражения (8) уравнение (7) примет вид

$$
\frac{d \varphi}{d x}=-\sqrt{\frac{8 n^{0} k T}{\varepsilon \varepsilon_{0}}} \operatorname{sh}\left[\frac{z e \varphi(x)}{2 k T}\right] .
$$

После интегрирования уравнения (9) с учетом граничных условий (4) получим

$$
\varphi(x)=\frac{4 k T}{z e} \operatorname{Arth}\left(\operatorname{th}\left(z e \varphi_{0} / 4 k T\right) \exp (-x d)\right),
$$

где $d-$ характерная длина радиуса Дебая. Она определяется следующим образом [7]:

$$
d^{-1}=\left[\frac{\varepsilon \varepsilon_{0} k T}{\sum_{i=1}^{N} n_{i}^{0} z^{2} e^{2}}\right]^{1 / 2}
$$

Отметим, что на данном расстоянии экранируется поле заряженной частицы за счет накапливающегося вокруг нее заряда противоположного знака.

Мы предполагаем, что при помещении заряженного аминокислотного остатка белка в раствор с заданной ионной силой происходит экранирование заряда его сферы, т.е. уменьшение ее потенциала и увеличение ее эффективного радиуса за счет характерной длины радиуса Дебая.
Таким образом, из (10) получаем значения потенциала сферы на границе экранирования, а из выражения (11) радиус Дебая. Используя эти данные, мы находим новое значение заряда для каждой сферы, помещенной в солевой раствор.

Ниже приведены результаты расчетов характерного радиуса сферы, заряда на поверхности сферы, моделируемый аминокислотный остаток и его электростатический потенциал в солевом растворе при различной ионной силе с учетом эффекта экранирования (табл. 1)

Проведенные ранее эксперименты [8] свидетельствуют о том, что взаимодействие белковых молекул обусловлено потенциальной энергией электростатического взаимодействия. Таким образом нами была также рассмотрена задача электростатического взаимодействия между белковыми молекулами и их укороченных аналогов в рамках классической электростатической теории.

Опишем кратко физическую модель электростатического взаимодействия между аминокислотными последовательностями различных белков. Каждая аминокислота представлена в виде равномерно заряженной сферы со своим значением радиуса. В данной модели мы не учитываем сворачивания полипептидной цепи белка в правильную пространственную структуру. Белок мы представляем как свободно-сочлененную полиаминокислотную последовательность. Отметим, что более подробное описание модели приводится в [9]. Далее решается задача электростатического взаимодействия двух заряженных шаров [10-12] с учетом эффекта экранирования в солевом растворе и, полученные в результате решения этой задачи, значения потенциальной энергии электростатического взаимодействия между соответствующими аминокислотными остатками записываются в соответствующую матрицу.

Для анализа биохимических процессов используем понятие числа обусловленности, которое будет характеризовать в данной физической постановке степень устойчивости конфигурации биологического комплекса. При этом для выбора более устойчивого биохимического соединения между белками мы выбираем матрицу потенциальной энергии электростатического взаимодействия с наименьшим значением числа обусловленности.

\section{2. Численное моделирование взаимодействия аминокислотных остатков}

\section{1. Моделирование пошагового образования гистоновых димеров (Н3-H4) и (H2A-H2B)}

В данной разделе нами были выполнены расчеты и проведен анализ устойчивости образования димеров $(\mathrm{H} 3-\mathrm{H} 4)$ и $(\mathrm{H} 2 \mathrm{~A}-\mathrm{H} 2 \mathrm{~B})$ при различных концентрациях ионной силы одновалентной соли путем анализа их 
Таблица 1. Результаты расчетов радиуса сферы, заряда для аминокислотных остатков в солевом растворе

\begin{tabular}{|c|c|c|c|c|c|c|}
\hline$n^{0}, \mathrm{~mol}$ & Аминокислотные остатки & $\mathrm{R}$ & $\mathrm{D}$ & $\mathrm{E}$ & $\mathrm{H}$ & K \\
\hline 0.01 & $\begin{array}{c}\text { Радиус сферы, nm } \\
\text { Заряд, С }\end{array}$ & $\begin{array}{c}0.8308 \\
1.306 \cdot 10^{-19}\end{array}$ & $\begin{array}{c}0.86 \\
0.85 \cdot 10^{-19}\end{array}$ & $\begin{array}{c}0.735 \\
1.01 \cdot 10^{-19}\end{array}$ & $\begin{array}{c}0.759 \\
1.07 \cdot 10^{-19}\end{array}$ & $\begin{array}{c}0.758 \\
1.08 \cdot 10^{-19}\end{array}$ \\
\hline 0.05 & То же & $\begin{array}{c}0.857 \\
1.275 \cdot 10^{-19}\end{array}$ & $\begin{array}{c}0.713 \\
0.867 \cdot 10^{-19}\end{array}$ & $\begin{array}{c}0.762 \\
0.998 \cdot 10^{-19}\end{array}$ & $\begin{array}{c}0.78 \\
1.04 \cdot 10^{-19}\end{array}$ & $\begin{array}{c}0.785 \\
1.062 \cdot 10^{-19}\end{array}$ \\
\hline 0.1 & $\ll \gg$ & $\begin{array}{c}0.878 \\
1.251 \cdot 10^{-19}\end{array}$ & $\begin{array}{c}0.734 \\
0.854 \cdot 10^{-19}\end{array}$ & $\begin{array}{c}0.783 \\
0.981 \cdot 10^{-19}\end{array}$ & $\begin{array}{c}0.801 \\
1.03 \cdot 10^{-19}\end{array}$ & $\begin{array}{c}0.806 \\
1.0442 \cdot 10^{-19}\end{array}$ \\
\hline 0.25 & $\ll \gg$ & $\begin{array}{c}0.918 \\
1.203 \cdot 10^{-19}\end{array}$ & $\begin{array}{c}0.774 \\
0.825 \cdot 10^{-19}\end{array}$ & $\begin{array}{c}0.823 \\
0.946 \cdot 10^{-19}\end{array}$ & $\begin{array}{c}0.841 \\
0.993 \cdot 10^{-19}\end{array}$ & $\begin{array}{c}0.846 \\
1.00 \cdot 10^{-19}\end{array}$ \\
\hline 0.5 & $\ll \gg$ & $\begin{array}{c}0.963 \\
1.14 \cdot 10^{-19}\end{array}$ & $\begin{array}{c}0.819 \\
0.791 \cdot 10^{-19}\end{array}$ & $\begin{array}{c}0.868 \\
0.905 \cdot 10^{-19}\end{array}$ & $\begin{array}{c}0.886 \\
0.949 \cdot 10^{-19}\end{array}$ & $\begin{array}{c}0.891 \\
0.961 \cdot 10^{-19}\end{array}$ \\
\hline 0.75 & $\ll \gg$ & $\begin{array}{c}0.998 \\
1.1 \cdot 10^{-19}\end{array}$ & $\begin{array}{c}0.854 \\
0.764 \cdot 10^{-19}\end{array}$ & $\begin{array}{c}0.903 \\
0.873 \cdot 10^{-19}\end{array}$ & $\begin{array}{c}0.921 \\
0.914 \cdot 10^{-19}\end{array}$ & $\begin{array}{c}0.926 \\
0.926 \cdot 10^{-19}\end{array}$ \\
\hline 1.0 & $\ll \gg$ & $\begin{array}{c}1.027 \\
1.062 \cdot 10^{-19}\end{array}$ & $\begin{array}{c}0.883 \\
0.74 \cdot 10^{-19}\end{array}$ & $\begin{array}{c}0.923 \\
0.844 \cdot 10^{-19}\end{array}$ & $\begin{array}{c}0.95 \\
0.884 \cdot 10^{-19}\end{array}$ & $\begin{array}{c}0.955 \\
0.895 \cdot 10^{-19}\end{array}$ \\
\hline 1.25 & $\ll \gg$ & $\begin{array}{c}1.053 \\
1.02 \cdot 10^{-19}\end{array}$ & $\begin{array}{c}0.909 \\
0.719 \cdot 10^{-19}\end{array}$ & $\begin{array}{c}0.958 \\
0.819 \cdot 10^{-19}\end{array}$ & $\begin{array}{c}0.976 \\
0.857 \cdot 10^{-19}\end{array}$ & $\begin{array}{c}0.981 \\
0.868 \cdot 10^{-19}\end{array}$ \\
\hline 1.5 & $\ll \gg$ & $\begin{array}{c}1.0767 \\
0.996 \cdot 10^{-19}\end{array}$ & $\begin{array}{c}0.9327 \\
0.700 \cdot 10^{-19}\end{array}$ & $\begin{array}{c}0.9817 \\
0.796 \cdot 10^{-19}\end{array}$ & $\begin{array}{c}0.9997 \\
0.833 \cdot 10^{-19}\end{array}$ & $\begin{array}{c}1.0047 \\
0.843 \cdot 10^{-19}\end{array}$ \\
\hline 1.75 & $\ll \gg$ & $\begin{array}{c}1.09 \\
0.968 \cdot 10^{-19}\end{array}$ & $\begin{array}{c}0.954 \\
0.681 \cdot 10^{-19}\end{array}$ & $\begin{array}{c}1.00 \\
0.775 \cdot 10^{-19}\end{array}$ & $\begin{array}{c}1.02 \\
0.81 \cdot 10^{-19}\end{array}$ & $\begin{array}{c}1.03 \\
0.76 \cdot 10^{-19}\end{array}$ \\
\hline 2.00 & $\ll \gg$ & $\begin{array}{c}1.118 \\
0.941 \cdot 10^{-19}\end{array}$ & $\begin{array}{c}0.974 \\
0.649 \cdot 10^{-19}\end{array}$ & $\begin{array}{c}1.023 \\
0.755 \cdot 10^{-19}\end{array}$ & $\begin{array}{c}1.041 \\
0.789 \cdot 10^{-19}\end{array}$ & $\begin{array}{c}1.046 \\
0.798 \cdot 10^{-19}\end{array}$ \\
\hline
\end{tabular}

Таблица 2. Числа обусловленности матриц потенциальной энергии электростатического взаимодействия для димера (Н3-Н4)

\begin{tabular}{c|c|c|c|c|c|c|c}
\hline$n^{0}, \mathrm{~mol}$ & 0.01 & 0.05 & 0.1 & 0.25 & 0.5 & 0.75 & 1.0 \\
\hline cond & $3.8 \cdot 10^{11}$ & $4.2 \cdot 10^{11}$ & $1.05 \cdot 10^{11}$ & $2.66 \cdot 10^{9}$ & $2.02 \cdot 10^{9}$ & $1.55 \cdot 10^{9}$ & $2.73 \cdot 10^{8}$
\end{tabular}

Таблица 3. Числа обусловленности матриц потенциальной энергии электростатического взаимодействия для димера (Н2А-Н2В)

\begin{tabular}{c|c|c|c|c|c|c|c}
\hline$n^{0}, \mathrm{~mol}$ & 0.01 & 0.05 & 0.1 & 0.25 & 0.5 & 0.75 & 1.0 \\
\hline cond & $2.8 \cdot 10^{10}$ & $6.19 \cdot 10^{10}$ & $8.79 \cdot 10^{8}$ & $9.06 \cdot 10^{8}$ & $1.55 \cdot 10^{9}$ & $9.12 \cdot 10^{10}$ & $3.12 \cdot 10^{8}$
\end{tabular}

потенциальной энергии электростатического взаимодействия. Для выполнения расчетов были сформированы блочные матрицы потенциальной энергии взаимодействия аминокислотных остатков, которую составили соответствующие матрицы взаимодействия различных комбинаций белков: (Н3-H4), (H4-H3) и (Н2А-H2B), (Н2B-H2А). Результаты выполненных расчетов приведены в табл. 2 и 3.

Примечание: cond - число обусловленности матрицы потенциальной энергии электростатического взаимодействия.
Следует отметить, что результаты ранее выполненных экспериментальных работ [13] показали, что образование димера (Н2A-H2B) происходит за счет большого количества контактов между аминокислотными остатками двух гистонов, причем область наименьшего взаимодействия приходится на гибкие концы молекул [13]. В [14] указывается, что взаимодействия димеров $(\mathrm{H} 2 \mathrm{~A}-\mathrm{H} 2 \mathrm{~B})$ и $(\mathrm{H} 3-\mathrm{H} 4)$ происходят по большому количеству аминокислотных остатков в центральном участке мономеров Н2А и Н2B, Н3 и Н4.

Численные результаты расчетов и анализ матрицы потенциальной энергии электростатического взаимодей- 
ствия белков Н2А и Н2В, Н3 и $\mathrm{H} 4$ показали, что с увеличением концентрации одновалентной соли в растворе происходит стабилизация димеров (H3-H4) и $(\mathrm{H} 2 \mathrm{~A}-\mathrm{H} 2 \mathrm{~B})$ при их образовании из мономеров Н3 и $\mathrm{H} 4$, Н2А и Н2В соответственно.

При более подробном рассмотрении результатов, приведенных в табл. 3, видно, что наблюдается значительное увеличение числа обусловленности для димеpa (Н2A-H2B) при концентрации одновалентной соли $0.75 \mathrm{~mol}$ и резкое уменьшение числа обусловленности при следующем значении концентрации 1 mol. Мы предполагаем, что данный эффект связан с изменением структуры молекул мономеров при связывании их в димер, т.е. при вращении звеньев молекул вокруг связи $[15,16]$.

Числа обусловленности матрицы потенциальной энергии электростатического взаимодействия могут периодически изменяться в зависимости от транс-конформации (скрещенная конформация) и цис-конформации (заслоненные конформации, т.е. когда проекции связей на плоскость совпадают). При этом минимуму числа обусловленности потенциальной энергии будут отвечать транс-конформации, а максимум - цис-конформации.

Наша модель дает результат одного из возможных вариантов формирования биологического комплекса димеров из мономеров.

Как мы видим из результатов, приведенных в табл. 3, димер (Н2A-H2В) является более устойчивым соединением, чем димер (H3-H4). Данный результат согласуется с ранее выполненной экспериментальной работой [2]. Отметим, что предыдущие исследования показали, что оба гетеродимера $(\mathrm{H} 3-\mathrm{H} 4)$ и $(\mathrm{H} 2 \mathrm{~A}-\mathrm{H} 2 \mathrm{~B})$ стабилизируются при увеличении ионной силы раствора $[2,14,17,18]$.

\section{2. Образование гистонового тетрамера $(\mathrm{H} 3-\mathrm{H} 4)_{2}$}

Нами было выполнено моделирование образования гистонового тетрамера $(\mathrm{H} 3-\mathrm{H} 4)_{2}$ в растворах с различной концентрацией одновалентной соли.

Согласно ранее выполненным экспериментальным работам, за взаимодействие двух одинаковых димеров (H3-H4) при образовании тетрамера отвечают несколько аминокислотных остатков гистонов Н3 [2]. Нами была составлена блочная матрица потенциальной энергии взаимодействия аминокислотных остатков, которую составили соответствующие матрицы образования различных комбинаций белков: (H3-H3), (H3-H4), (H4-H3), ( $\mathrm{H} 4-\mathrm{H} 4)$.

Мы проанализировали полученную блочную матрицу потенциальной энергии взаимодействия аминокислотных остатков и выбрали в ней участок с наибольшими значениями потенциальной энергии электростатического взаимодействия, отбросив участки матрицы, в которых значения потенциальной энергии были минимальными. В результате мы получили новую матрицу
Таблица 4. Числа обусловленности матриц потенциальной энергии электростатического взаимодействия для тетрамера $(\mathrm{H} 3-\mathrm{H} 4)_{2}$.

\begin{tabular}{c|c|c|c|c|c}
\hline$n^{0}, \mathrm{~mol}$ & 0.1 & 0.25 & 0.5 & 0.75 & 1.0 \\
\hline cond & $6.87 \cdot 10^{11}$ & $7.66 \cdot 10^{8}$ & $2.84 \cdot 10^{10}$ & $3.68 \cdot 10^{9}$ & $8.77 \cdot 10^{9}$
\end{tabular}

Таблица 5. Числа обусловленности матриц потенциальной энергии электростатического взаимодействия для гексамера $(\mathrm{H} 3-\mathrm{H} 4)_{2}(\mathrm{H} 2 \mathrm{~A}-\mathrm{H} 2 \mathrm{~B})$

\begin{tabular}{c|c|c|c|c|c}
\hline$n^{0}, \mathrm{~mol}$ & 1.0 & 1.25 & 1.5 & 1.75 & 2.0 \\
\hline cond & 13900 & 11700 & 915 & 611 & 3250
\end{tabular}

потенциальной энергии электростатического взаимодействия при этом в новую матрицу полностью вошли взаимодействия гистонов $(\mathrm{H} 3-\mathrm{H} 3)$ и частично $(\mathrm{H} 4-\mathrm{H} 4)$. Результаты выполненных расчетов приведены в табл. 4.

Как видно из данных табл. 4, с увеличением ионной силы происходит увеличение силы взаимодействия между димерами (H3-H4) и (H3-H4).

Отметим, что результаты таблиц 2, 3 и 4 свидетельствуют о том, что димеры $(\mathrm{H} 2 \mathrm{~A}-\mathrm{H} 2 \mathrm{~B})$ и $(\mathrm{H} 3-\mathrm{H} 4)$ в растворах с ионной силой от $0.1 \mathrm{~mol}$ до $1 \mathrm{~mol}$ являются более стабильными биологическими комплексами, чем тетрамер $(\mathrm{H} 3-\mathrm{H} 4)_{2}$ при тех же концентрациях раствора одновалентной соли.

Данный результат согласуется с работой [2], в которой указывается на менее устойчивое объединение двух димеров (Н3-H4) в тетрамер, чем связывание мономеров Н3, Н4 и Н2А, Н2B в димеры.

\section{3. Образование гексамера и октамера}

Нами было выполнено моделирование сборки гексамера $(\mathrm{H} 3-\mathrm{H} 4)_{2}(\mathrm{H} 2 \mathrm{~A}-\mathrm{H} 2 \mathrm{~B}) \quad$ и октамера $(\mathrm{H} 3-\mathrm{H} 4)_{2}(\mathrm{H} 2 \mathrm{~A}-\mathrm{H} 2 \mathrm{~B})_{2}$ при различных концентрациях раствора одновалентной соли. Отметим, что в [3] указывается на многочисленные контакты между гистонами в данных структурах.

Нами была составлена блочная матрица потенциальной энергии взаимодействия аминокислотных остатков, которую составили соответствующие матрицы образования различных комбинаций белков: (Н3-H3), (Н3-H4), $(\mathrm{H} 4-\mathrm{H} 4), \quad(\mathrm{H} 2 \mathrm{~A}-\mathrm{H} 2 \mathrm{~B}), \quad(\mathrm{H} 2 \mathrm{~A}-\mathrm{H} 2 \mathrm{~A}), \quad(\mathrm{H} 2 \mathrm{~B}-\mathrm{H} 2 \mathrm{~B})$, (H2A-H3), (H2A-H4), (Н2B-H3), (Н2B-H4). Анализ таких матриц приведен в табл. 5 и 6.

Из данных табл. 5 и 6 видно, что увеличение стабильности биологических комплексов происходит по мере увеличения ионной силы, при этом в растворе с концентрацией $2 \mathrm{~mol}$ гексамер может достичь более устойчивого положения при присоединении еще одного 
Таблица 6. Числа обусловленности матриц потенциальной энергии электростатического взаимодействия для октамера $\mathrm{H} 3-\mathrm{H} 4)_{2}(\mathrm{H} 2 \mathrm{~A}-\mathrm{H} 2 \mathrm{~B})_{2}$

\begin{tabular}{c|c|c|c|c|c}
\hline$n^{0}, \mathrm{~mol}$ & 1.0 & 1.25 & 1.5 & 1.75 & 2.0 \\
\hline cond & 20400 & 2230 & 634 & 463 & 385
\end{tabular}

димера (Н2A-H2B) и переходом таким образом в октамер.

Выполненные ранее работы $[4,19]$ так же отмечают стабильность гексамера и октамера при высоких значениях ионной силы и последующей диссоциацией комплексов до тетрамеров $(\mathrm{H} 3-\mathrm{H} 4)_{2}$ и димеров $(\mathrm{H} 2 \mathrm{~A}-\mathrm{H} 2 \mathrm{~B})$ при уменьшении ионной силы раствора.

\section{Заключение}

Математическое моделирование, выполненное в настоящей работе на биологических объектах с использованием гистоновых белков Н2А, Н2B, Н3 и Н4, продемонстрировало возможность прогнозирования устойчивости биологического комплекса для случая in vitro в растворах с различной ионной силой.

Анализ выполненных расчетов показал, что формированию более устойчивых биологических комплексов отвечают различные концентрации одновалентной соли растворов.

Введенный нами критерий (число обусловленности) позволяет прогнозировать уменьшение и увеличение силы связывания гистоновых белков при образовании гистонового октамера на всех стадиях образования комплекса с учетом экранирования заряда заряженных аминокислотных остатков белков.

Так, при образовании димеров (Н3-H4) из мономеров, увеличение концентрации одновалентной соли от 0.01 до $1 \mathrm{~mol}$ приводит к образованию более стабильного комплекса.

При образовании димера (Н2A-H2B), как мы видим из табл. 2 и 3 , увеличение концентрации одновалентной соли также приводит к увеличению стабилизации комплекса, за исключением результата, полученного нами для концентрации $0.75 \mathrm{~mol}$.

Данное увеличение числа обусловленности мы объясняем тем, что наша модель не учитывала конформации гистонов при образовании биологических комплексов, когда могут осуществляться как цис-конформации, так и транс-конформации. Анализ формирования тетрамера $(\mathrm{H} 3-\mathrm{H} 4)_{2}$ указывает на увеличение стабилизации биологического комплекса при увеличении ионной силы раствора с 0.1 до $1.0 \mathrm{~mol}$, а также на то, что тетрамерный комплекс гораздо менее стабилен, чем димеры (H3-H4) и (Н2A-H2B), поскольку осуществление связывания димеров (H3-H4) в тетрамер осуществляется за счет небольшого количества взаимодействующих аминокислот между одинаковыми белками Н3 в отличие от механизма формирования димеров (Н2A-H2B) и (Н3-H4), у которых связывание мономеров в димеров осуществляется за счет большого количества аминокислотных остатков центральных участков молекулы.

Данные результаты хорошо согласуются с ранее выполненными экспериментальными работами $[2,14,18]$. Моделирование формирования гексамера и октамера мы проводили с учетом концентрации растворов одновалентной соли в пределах от 1.0 до $2.0 \mathrm{~mol}$.

Полученные результаты демонстрируют увеличение стабилизации комплекса октамера при концентрации одновалентной соли до $2.0 \mathrm{~mol}$. При этом увеличение стабилизации гексамера происходит от величины концентрации одновалентной соли 1.0 до $1.75 \mathrm{~mol}$, в пределах выполненных нами расчетов. При концентрации $2.0 \mathrm{~mol}$, как вы видим из табл. 5, устойчивость комплекса гексамера несколько снижается. При присоединении к данному комплексу четвертого димера (H2A-H2B) гексамер переходит в более устойчивое состояние октамера с восемью гистоновыми белками, что также согласуется с работами $[4,19]$, где экспериментально исследовался вопрос, связанный с увеличением стабильности комплексов гексамера и октамера в растворах с большой ионной силой.

\section{Список литературы}

[1] Iwasaki W., Miya Y., Horikoshi N., Osakabe A., Taguchi H., Tachiwana H., Shibata T., Kagawa W., Kurumizaka H. // FEBS Open Bio. 2013 Vol. 3. P. 363-369.

[2] Banks D.D., Gloss L.M. // Biochemistry. 2003. Vol. 42. N 22. P. 6827-6839.

[3] Marico-Ramírez L., Kann M.G., Shoemaker B.A., Landsman D. // Expert Rev. Proteomics. 2005. Vol. 2. N 5. P. 719-729.

[4] Dias R., Lindman B. DNA Interactions with Polymers and Surfactans. John Wiley \& Sons, Inc. 2008.

[5] Духин С.C. Диэлектрические явления и двойной слой в дисперсных системах и полиэлектролитах. Киев: Наукова думка, 1972.

[6] Keith B. Oldham // J. Electroanalyt. Chem. 2008. Vol. 613. P. 131-138.

[7] Masliyah J.H., Bhattacharjee S. Electrokinetic and Colloid Transport Phenomena. John Wiley \& Sons, Inc. 2006.

[8] Fenley A.T., Adams D.A., Onufriev A.V. // Biophys. J. 2010. Vol. 99. P. 1577-1585.

[9] Куликов К.Г., Кошлан Т.В. // ЖТФ. 2016. Т. 86. Вып. 10. C. 131-138.

[10] Саранин В.А. // УФН. 1999. Т. 169. № 4. С. 453-458.

[11] Саранин B.A. // УФН. 2002. Т. 172. № 12. С. 1449-1454.

[12] Смайm В. Электростатика и электродинамика. М.: ИЛ, 1954.

[13] Moriwaki Y., Yamane T., Ohtomo H., Ikeguchi M., Kurita J., Sato M., Nagadoi A., Shimojo H., Nishimura Y. // Scientific Reports. 2016. Vol. 6. N 24999. P. 1-11.

[14] Karantza V., Freire E., Moudrianakis E.N. // Biochemistry. 2001. Vol. 40. N 43. P. 13114-13123. 
[15] Волькенштейн В.M. Молекулы и жизнь. М.: Наука, 1965.

[16] Семчиков Ю.Д. Высокомолекулярные соединения. М.: Академия, 2010.

[17] Khrapunov S.N., Dragan A.I., Protas A.F., Berdyshev G.D. // Biochim. Biophys. Acta. 1984. Vol. 787. N 1. P. 97-104.

[18] Karantza V., Freire E., Moudrianakis E.N. // Biochemistry. 1996. Vol. 35. N 6. P. 2037-2046.

[19] Eickbush T.H., Moudrianakis E.N. // Biochemistry. 1978. Vol. 17. N 23. P. 4955-4964. 